\title{
Point forecasts of mortality rates in Malaysia: A comparison of principal component methods
}

\author{
Wan Zakiyatussariroh Wan Husin ${ }^{1}$, Nazatul Shahreen Zainal Abidin ${ }^{2}$ \\ ${ }^{1}$ Faculty of Computer and Mathematical Sciences, Universiti Teknologi MARA Cawangan Kelantan, Malaysia \\ ${ }^{2}$ Academy of Language Studies, Universiti Teknologi MARA Cawangan Kelantan, Malaysia
}

\begin{tabular}{l} 
Article Info \\
\hline Article history: \\
Received Jan 3, 2020 \\
Revised Mar 15, 2020 \\
Accepted Apr 7, 2020 \\
\hline
\end{tabular}

Keywords:

Age-specific death rates

Forecast accuracy

Mortality rates

Point forecast

Principal component method

\begin{abstract}
The problem of forecasting mortality has been widely discussed and is being considered as an important issue among actuaries and demographers. Using age and sex-specific mortality data in Malaysia, we compare and evaluate the point forecast accuracy of six principal component methods. These six methods are the Lee-Carter (LC) method, the unadjusted LC method (LCnone), the Lee-Miller (LM) variant, the Booth-Mandonald-Smith variant (BMS) and the two versions of the Hyndman-Ullah (HU). The overall performance of all methods was evaluated using out-sample forecasts based on the six error measures of three different data spanning with three different step-ahead forecasts. The data set consists of annual age-specific death rates (ASDR) for 17 age groups for both genders for the years 1980 to 2011. Results show that when the fitted period is longer, the LM method exhibits a better fit in Malaysian male ASDR and the HU6 method attains a better fit in Malaysian female ASDR. When the fitted period becomes shorter, the BMS method outperforms for male and HU1 for female ASDR. However, for overall performance, the LM method consistently outperforms others for Malaysian male ASDR and the LM and HU methods are the best good of fit for Malaysia ASDR.
\end{abstract}

Copyright $(0) 2020$ Institute of Advanced Engineering and Science. All rights reserved.

\section{Corresponding Author:}

Wan Zakiyatussariroh Wan Husin,

Faculty of Computer and Mathematical Sciences,

Universiti Teknologi MARA Cawangan Kelantan,

18500 Bukit Ilmu, Machang, Kelantan, Malaysia.

Email: wanzh@uitm.edu.my

\section{INTRODUCTION}

Mortality forecasting is of general interest as it becomes the most primary attribute of human life and an important element in predicting population, household and related forecast forms and it is fundamental in social and economic planning. The improvement of mortality rates in many countries in the world has a major impact on costs associated with living longer due to mortality and morbidity risks [1]. Projections of mortality improvements have, therefore, become increasingly important among actuaries and demographers since mortality has improved considerably faster than had been predicted before. Considerable attention has been paid to methods for forecasting mortality in recent years. Many recent works have grown out of the Lee-Carter (LC) method [2]. The LC method applied the concept of principal component method which transform attributes of data set into new set of uncorrelated attributes in reducing the dimensionality while maintaining as much of the variability of the data set as possible [3]. The LC method assumes that the log-death rates time series shares one common trend that is explained by the first principal component term which is represented by mortality index. The strength of the LC method lies on its simplicity and robustness in the context of linear trends in age-specific death rates (ASDR) [4]. This method has been regarded as among the most excellent approach currently in forecasting mortality rates because it yields statistically more precise results compared to other approaches $[5,6]$. 
Previously, numerous variants of the LC method have been made in the search for the most appropriate approach to produce better forecasts. These include Lee-Miller (LM) variant [7], LC without adjustment (LCnone) [8] and Booth-Maindonald-Smith (BMS) [9] variant. Besides that, there have also been several extensions of the LC method. Of these, the Hyndman-Ullah extension [10] has received increasing attention in the fields of demography and statistics. The Hyndman-Ullah extension, also known as HU method, combines the ideas of nonparametric smoothing, functional principal component regression and functional data analysis.

Studies on comparing and evaluating the accuracy of variants and extensions of the LC method for mortality forecasting have been conducted previously by numerous researchers [4-6, 11-15]. Some of the studies were conducted using mortality data of the origin country of the researchers while some used mortality data from several countries. As highlighted by Cairns et al. [16], forecasters should not assume that the same stochastic methods for a data set will be suitable for others. Therefore, the process of selecting the appropriate methods for a period and a country should not be by-passed. Referring to this fact, in the case of Malaysia, there are numerous efforts in forecasting Malaysia mortality using the LC method such as a study done by [17-19]. In 2014, Husin et al. [20] have evaluated the performance of the LC method, LM variant and BMS variant to Malaysia mortality. Then, in 2015, Husin et al. [21] investigated the feasibility of using the HU method in modeling and forecasting Malaysia mortality, then, identifying a better method between the original LC and HU method to Malaysia mortality data that can further be considered as an alternative method to forecast Malaysia mortality. On the other hand, Kamaruddin [22] compared the performance of HU method and BMS in forecasting mortality rate in Malaysia. While Shair [23] found that the HU method performed better compared to LC method in forecasting Malaysia mortality. However, a study done by [24] found opposite findings with [23] when evaluating the performance of LC and HU methods. In addition, in most countries, the mortality rates among females are generally lower than males at almost all ages $[25,26]$. Thus, this paper extended the studies of previous researchers by comparing and evaluating the performance of the LC method and its variants together with HU method to Malaysia mortality separated by gender. Three different mortality data spanning were used in evaluating the forecast performance of the methods.

\section{RESEARCH METHOD}

This section describes the forecasting methods and evaluation procedure applied in this study. There are two simple steps applied in evaluations of the forecasting methods. The first step involved insample models estimation using training data and the second step was model evaluation which was based on out-of-sample or forecast evaluation. Model evaluations focused on checking the performance of the models based on overall forecast accuracy. Then, a comparison was conducted among the families of each models; LC and its variants and $\mathrm{Hu}$ method using six error measures.

\subsection{Forecasting method}

There is a wide range of frequently used forecasting methods in forcasting mortality. However, the focus of this study is on the mortality forecasting methods which are based on principal components only. The methods are the original LC method [2], the three variants of LC which are LM [7], LCnone [8] and BMS [9] and the extension of LC method, HU method [10].

\subsubsection{Lee-Carter (LC) method}

The LC method is a method involving a single time-varying parameter which explains the linear relationship among logarithm of ASDR, $m_{x, t}$ and two factors that are age $x ; x=1, \ldots, N$ and year $t ; t=1, \ldots$, $T$. The model is presented as

$$
\ln \left(m_{x, t}\right)=\alpha_{x}+\beta_{x} k_{t}+\varepsilon_{x, t}
$$

where $m_{x, t}$ is the observed ASDR at age $x$ in year $t, \alpha_{x}$ is a fixed age effect that is an average of observed log ASDR at age $x, k_{t}$ is a time-trend index of general mortality level (mortality index) and $\beta_{x}$ is an age-specific impact of $k_{t}$ for a particular time $t$. The term $\beta_{x}$ is assumed to be time invariant. The term $\varepsilon_{x, t}$ is random error with means 0 and variance, $\sigma^{2}$. The time dependent $k_{t}$ captures the time trend in mortality rates on the logarithmic scale for all ages and represents the average deviation from $\alpha_{x}$ of the actual pattern of mortality. The $\alpha_{x}$ is calculated as the average observed $\log$ ASDR over time and $\beta_{x}$ and $k_{t}$ are estimated 
by a singular value decomposition (SVD). The LC method involved a two-stage estimation procedure. In the first stage, SVD is applied to estimate $\beta_{x}$ and $k_{t}$ that produces $\hat{\beta}_{x}$ and $\hat{k}_{t}$. In the second stage, $\hat{k}_{t}$ was adjusted to ensure equality between the observed and estimated number of deaths. The adjustment was made by refitting $\hat{k}_{t}$ to total number of deaths $D_{t}$. The adjusted $\hat{k}_{t}\left(\tilde{k}_{t}\right)$ is then extrapolated using ARIMA $(0,1$, 0 ) which is a random walk with drift model that can be presented as

$$
k_{t}=k_{t-1}+\theta+u_{t}
$$

where $\theta$ is the constant annual change in $k_{t}$ and $u_{t}$ is the error terms with $t=1, \ldots, T$. Forecasts of ASDR were obtained using extrapolated $k_{t}$ and fixed $\alpha_{x}$ and $\beta_{x}$ where the forecasted value of adjusted estimated $k_{t}$ and the estimated $\alpha_{x}$ and $\beta_{x}$ were substituted to the LC method of $m_{x, t}$ and then conveyed back to the original scale in order to forecast mortality rates. In this case, Lee and Carter [2] used fitted rates as the jump-off rates. Thus, the forecast of $\hat{m}_{x, T+1}$ is $\hat{m}_{x, T+1}=\exp \left(\hat{\alpha}_{x}+\hat{\beta}_{x} \tilde{k}_{T+1}\right)$.

\subsubsection{Lee-Carter without adjustment (LCnone) method}

Tuljapurkar et al. [8] became the first to modify the LC method without doing any adjustment on $k_{t}$ where the analysis of the mortality index $k_{t}$ is conducted based on the estimated $k_{t}$ without any adjustment when the LC method was fitted to the G7 countries (Canada, France, Germany, Italy, Japan, United Kingdom (UK) and United States (US)). Then, this approach was followed by [11]. This variant of LC method is known as LCnone.

\subsubsection{Lee-Miller (LM) method}

Lee and Miller [7] modified the LC method in three manners which are: i) reduce the fitting period; ii) the adjustment of $k_{t}$ involves fitting to life expectancy at birth $(\mathrm{e}(0))$ in year $t$ rather than fitting to total deaths in year $t$; and iii) the actual rates in jump-off year are used as the jump-off rates. These modifications were made after they found that the forecast that were based on the original LC method was biased with jump-off error because of the difference between the fitted rates for the last year of the fitting period and the actual rates in that year. They also revealed that the pattern of change in mortality rates was not constant over time as was assumed by the LC method and the adjustment of historical principal component scores resulted in a large estimation error.

\subsubsection{Booth-Maindonald-Smith (BMS) method}

Booth et al. [9] also modified the LC method in three manners. First, the fitting period was set based on statistical goodness-of-fit criteria under the assumption of a linear trend in the first principal component score $k_{t}$ and using deviance statistic $t$ to measure the goodness of fit. Second, the $k_{t}$ was adjusted by fitting the base method to the age distribution of deaths $D_{x, t}$ using the Poisson distribution rather than fit to total deaths $D_{t}$. Finally, the jump-off rate was set to be the fitted rates. These modifications were made after Booth et al. [9] found historical departures from linearity in Australian mortality declined where the linearity of $k_{t}$ did not hold over the fitting period. Booth et al. [4] concluded that the LM and BMS perform almost with the same performance.

\subsubsection{Hyndman-Ullah (HU) method}

Defining mortality rates for age $x$ in year $t$ to $m_{i}(x, t)$ where $x=1, \ldots, N$ and $t=1, \ldots, T$ and $y_{i}(x, t)=\ln m_{i}(x, t)$ the model for the HU method is written as

$$
\begin{aligned}
& y_{i}(x, t)=f_{i}(x, t)+\sigma_{i}(x, t) \varepsilon_{i, t}, \\
& f_{i}(x, t)=\alpha_{i}(x)+\sum_{j=1}^{J} k_{i, t, j} \beta_{i, j}(x)+e_{i}(x, t)
\end{aligned}
$$

where $f_{i}(x, t)$ is the smooth function of $x$ and $\sigma_{i}(x, t)$ is the smooth volatility function that varies with age $x$ in year $t$ and $\varepsilon_{t, i}$ is the errors having independent and identically distributed with standard normal distribution 
for every $i=1, \ldots, N$ and $t=1, \ldots, T$. As in the LC model, $\alpha_{i}(x)$ is the average pattern of mortality by age across years, estimated by $\hat{\alpha}_{i}(x)=\frac{1}{T} \sum_{t=1}^{T} f_{i}(x, t), \beta_{i, j}(x)$ is a set of orthonormal basis functions like $\beta_{x}$ in LC model that is a set of first $J$ principal components reflecting the sensitivity to time-varying index over age, $k_{i, t, j}$ is a set of uncorrelated principal component scores which are also explained as time series coefficients similar as $k_{t}$, in LC model representing the time varying index, $e_{i}(x, t)$ is the modeling error and $J<T$ is the number of principal components being extracted. For simplicity, the group index $i$ is ignored in the following analysis and $y(x, t)$ is rewritten as $y_{t}(x)$ and so on. Hence, the model in (3) and (4) is rewritten as follows:

$$
\begin{aligned}
& y_{t}(x)=f_{t}(x)+\sigma_{t}(x) \varepsilon_{t} \\
& f(x, t)=\alpha(x)+\sum_{j=1}^{J} k_{t, j} \beta_{j}(x)+e(x, t)
\end{aligned}
$$

The HU method utilises second and higher order principal components in capturing additional dimensions of change in mortality rates which are not explained by the first principal component. These additional dimensions were explained by the number of basis function $\beta_{j}(x), j=1, \ldots, J$ which is calculated using the principal components decomposition. In this study, the number of basis function $\beta_{j}(x)$ was set to $j=6$ (denoted as HU6), since according to Hyndman and Booth [27], $j=6$ principal components should be larger than any of the component really requires. In addition, the HU model with $j=1$ principal component was also assessed in order to examine the performance of the functional data model with respect to the original LC model which considered only one principal component. Univariate time series model i.e. ARIMA model was used to forecast the time-varying scores $k_{t, j}$. Combining the function in (5) and (6) yields:

$$
y_{t}(x)=\alpha(x)+\sum_{j=1}^{J} k_{t, j} \beta_{j}(x)+e_{t}(x)+\sigma_{t}(x) \varepsilon_{t},
$$

then, the $h$-step-ahead forecast of $y_{T+h}(x)$ is

$$
\hat{y}_{T+h}(x)=E\left[y_{T+h}(x)\right]=\hat{\alpha}(x)+\sum_{j=1}^{J} \tilde{k}_{T, j, h} \hat{\beta}_{j}(x)
$$

where $\tilde{k}_{T, j, h}$ is the $h$-step ahead forecast of $k_{T, j, h}$ through estimated of $\hat{k}_{1, j}, \ldots, \hat{k}_{T, j}$. Since $y_{i}(x, t)=\ln m_{i}(x, t)$ then the ASDR forecast is $\hat{m}_{T+h}(x)=\exp \left\{\hat{y}_{T+h}(x)\right\}$.

\subsection{Data description}

This study used ASDR in Malaysia separated by gender which were provided by the Department of Statistics Malaysia (DOSM). For each gender and each calendar year, the ASDR was calculated as $m_{x, t}=\frac{d_{x, t}}{p_{x, t}}$, where $m_{x, t}$ is observed ASDR, $d_{x, t}$ is the number of deaths for a group of $x$ ages and $p_{x, t}$ is

the observed population for a group of $x$ ages in year $t$ with $x=1, \ldots, N$ and in year $t ; t=1, \ldots, T$. The data consist of annual number of deaths and populations for 17 age groups that were organised into 5-year intervals; $0-4,5-9, \ldots, 80+$. Due to the limitation of data, this study only covered data from Peninsular Malaysia from the period 1980 to 2011 for both genders. The raw data were transformed by taking the natural logarithms to stabilise the high variance associated with high ASDR as shown in Figure 1 where the observed Malaysia ASDR from year 1980 to 2011 for both genders have fluctuated. During the past 32 years, Malaysia ASDR has decreased considerably in almost all age groups except for age 80+ and is much lower for female population than for the male population. The decline in ASDR for female population is steadier than those of the male population with data for the male population appears much noisier for most age groups. 

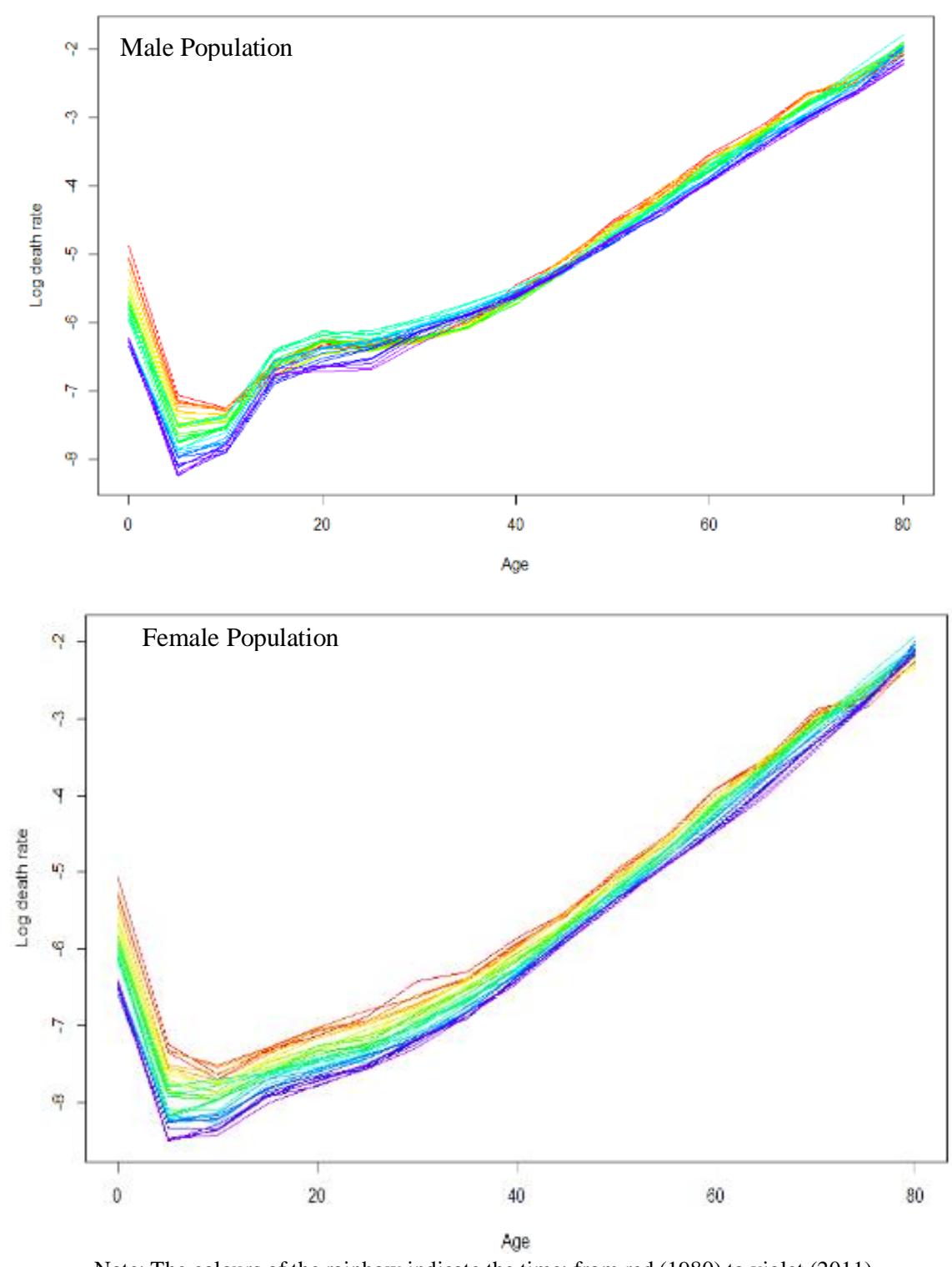

Note: The colours of the rainbow indicate the time; from red (1980) to violet (2011)

Figure 1. Malaysia log age-specific death rates over age group

\subsection{Evaluation procedure}

The evaluation procedure involved fitting all the six approaches to three training mortality data sets; 1980 - 2000, 1980 - 2005 and 1980 - 2009 to forecast periods 2001 - 2011 (eleven-step-ahead forecast), 2006 - 2011 (six-step-ahead forecast) and 2010 - 2011 (one-step-ahead forecast), respectively. Then, the forecast errors were determined by comparing the forecasted ASDR with the actual out-of-sample data. In particular, the six error measures were examined, namely root mean square error (RMSE), mean absolute percentage error (MAPE), mean absolute error (MAE), median absolute percentage error (MdAPE), median absolute error (MdAE) and geometric root mean square (GMRMSE). These measures were calculated based on the averaged errors over the age groups. The best performance model is based on minimum error. The equation of these error measures are as follows:

$$
\begin{aligned}
& R M S E=\sqrt{\operatorname{mean}\left(\boldsymbol{e}_{t}^{2}\right)} \\
& M A E=\operatorname{mean}\left|\boldsymbol{e}_{t}\right| \\
& M d A E=\text { median }\left|\boldsymbol{e}_{t}\right|
\end{aligned}
$$




$$
\begin{aligned}
& \text { GMRMSE }=\left(\prod_{t=1}^{T} \boldsymbol{e}_{t}^{2}\right)^{\frac{1}{2 T}} \\
& \text { MAPE = mean }\left|\frac{\boldsymbol{e}_{t}}{\boldsymbol{m}_{t}}\right| \times 100 \\
& \text { MdAPE }=\text { median }\left|\frac{\boldsymbol{e}_{t}}{\boldsymbol{m}_{t}}\right| \times 100
\end{aligned}
$$

\section{RESULTS AND ANALYSIS}

This study fitted all the six approaches to three training mortality data and produced three sets of point forecast accuracy for three different data spanning for the male population and female population. As mentioned previously, evaluation of the models was based on forecast accuracy which was measured by the error measure - the model with the smallest error measure is the best performing model compared to others. The findings were well illustrated for both genders using spider plot as in Figure 2, Figure 3 and Figure 4 for the three data span, respectively. For the fitted period 1980 - 2000 as shown in Figure Figure 2, it can be seen that almost all models have similar performance except RMSE and MdAE which show that the BMS outperformed others for male population which gives smaller errors, while the finding is opposite for female population, where most of the measures show the HU1 outperform others except RMSE and MAE shows HU6 is better performing. However, based on Figure 3, for fitted period $1980-2005$, it clearly shows that the LM model outperformed other models for all error measures for both male population and female population except for RMSE and MdAE of the female population. The same condition can be seen for fitted period 1980 - 2010 as shown in Figure 3 for male population in which LM also outperformed others. However, for this fitting period, in the case of female population, different error measures give difference findings. Hence, it is notable that, for the male population, the LM method is dominant compared to other methods in producing one-step-ahead forecasts (for fitting period 1980 - 2010) and six-step-ahead forecasts (for fitting period 1980 - 2005) using longer historical fitted period which gives the smallest error values for all error measures. However, for shorter fitted period with longer step-ahead forecasts, the BMS method outperformed others.

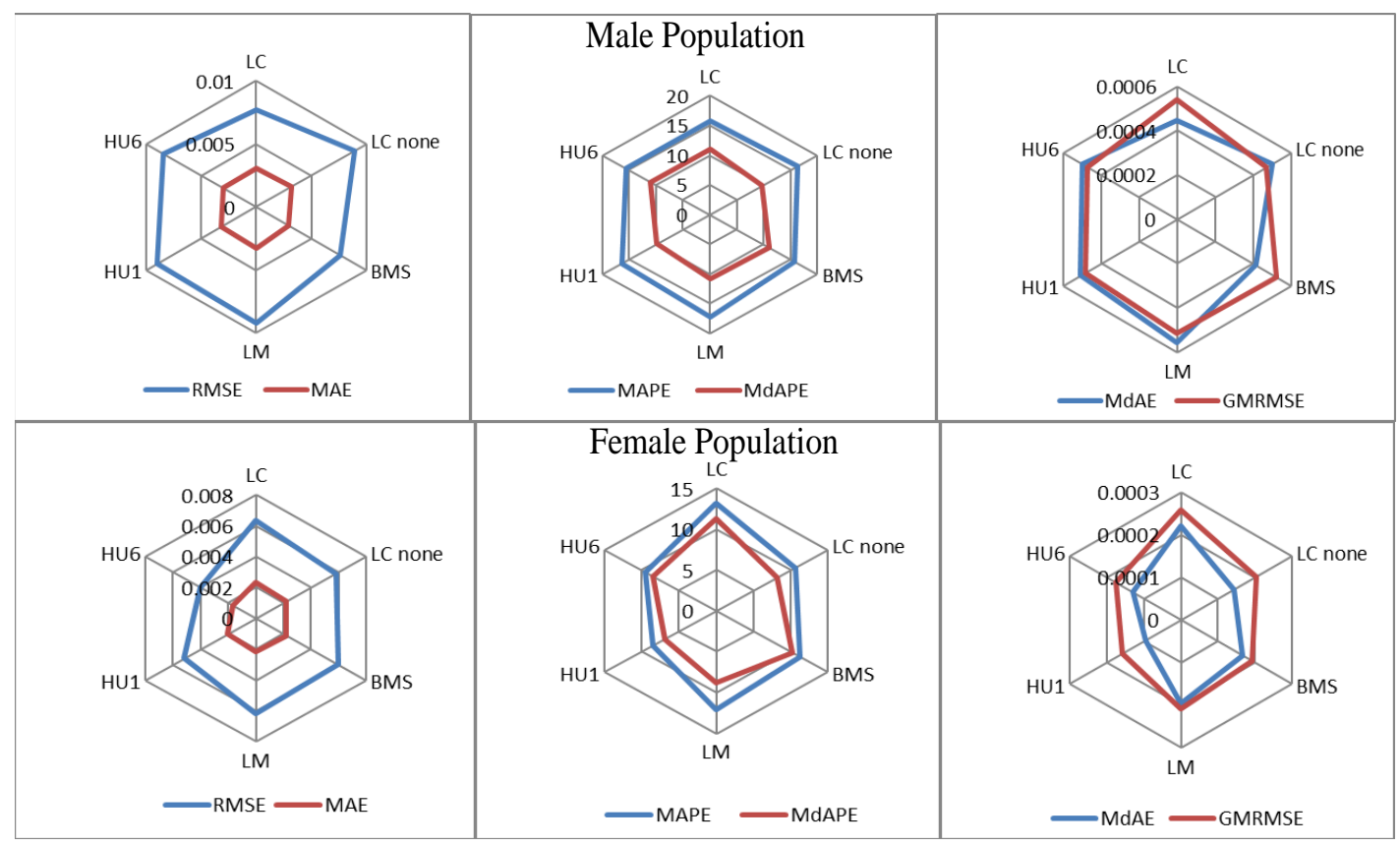

Figure 2. Forecast accuracy of ASDR based on fitted period $1980-2000$ 


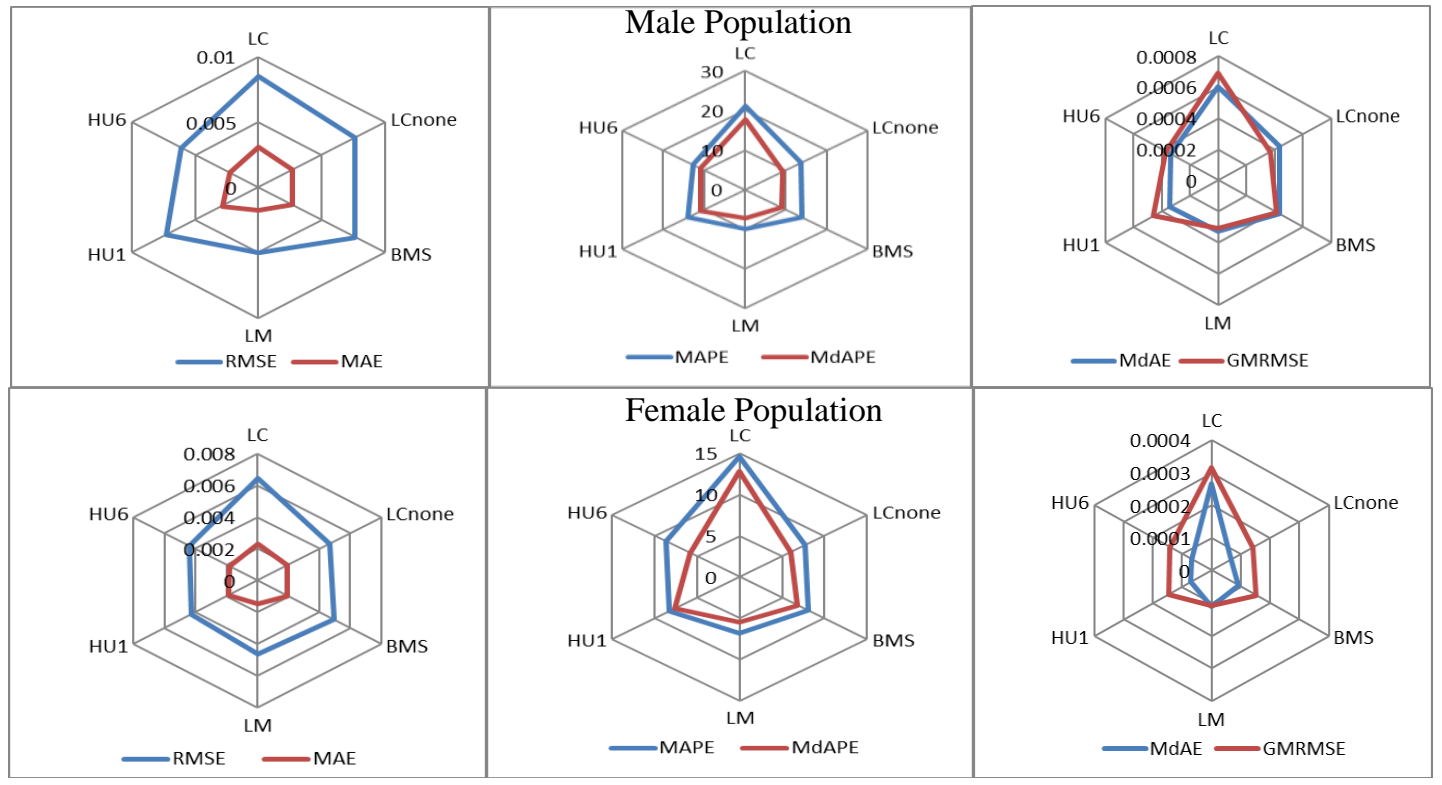

Figure 3. Forecast accuracy of ASDR based on fitted period $1980-2005$

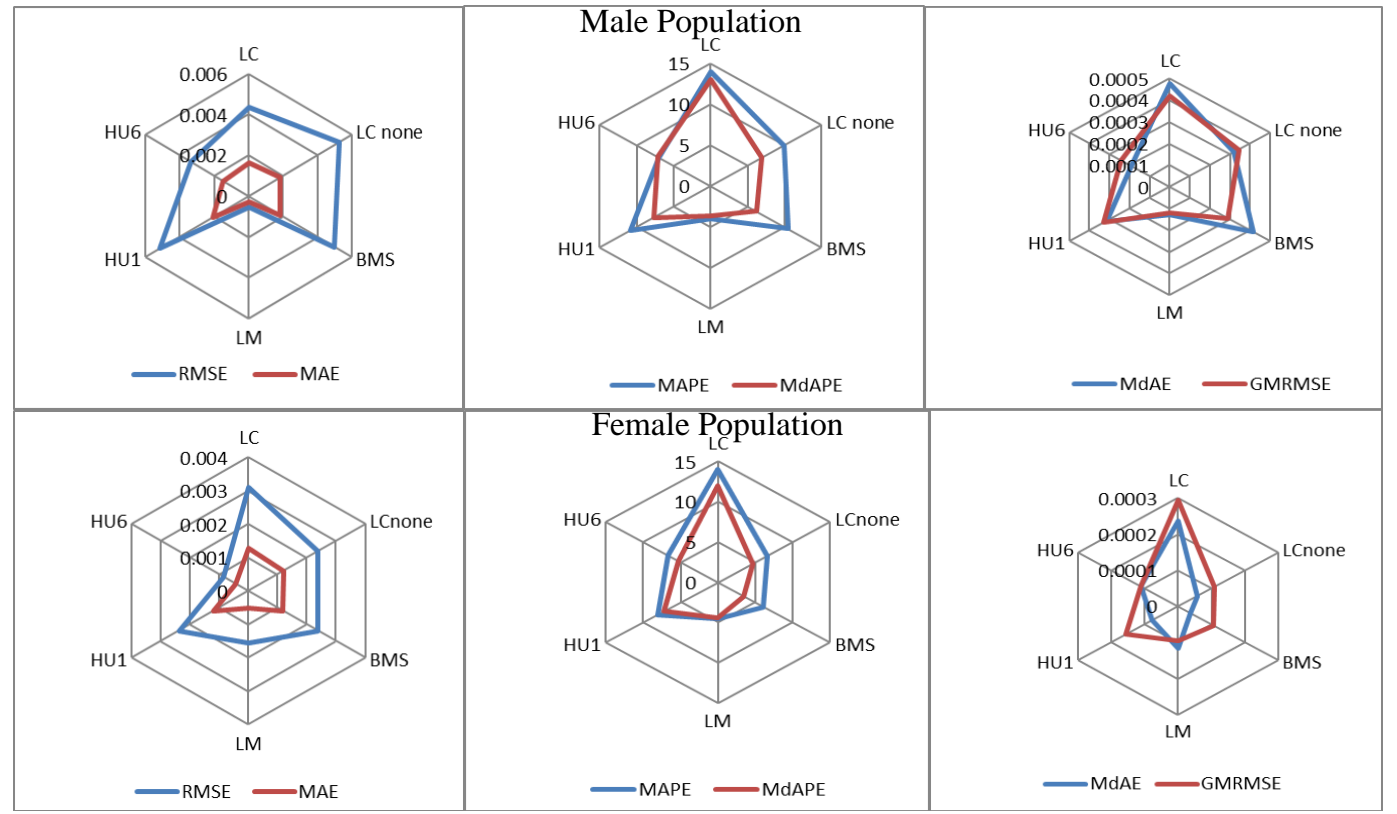

Figure 4. Forecast accuracy of ASDR based on fitted period 1980 - 2010

Nevertheless, opposite results were found in the data for female population, where none of the methods consistently outperformed the others. However, when the fitted period is shorter $(1980-2000)$, the HU method outperformed others with HU1 producing better forecasts compared to HU6. When the fitted period was increased by 5 years $(1980$ - 2005), the LM method was more consistent. However, in producing one-step-ahead forecasts for fitting period 1980 - 2010, almost all methods equally performed, with the LC method the worst. For fitted period 1980 - 2000 based on Figure 2, all error measures show that almost all models have similar performance for both male and female population except RMSE and GRMSE shows the BMS outperformed others for male population, and for female population, RMSE shows HU6, while MdAE shows HU1 outperformed others. However, based on Figure 3, for fitted period $1980-2005$, it clearly shows that the LM method outperformed other models for almost all error measures for both male population and female population. Different findings can be seen for fitted period 1980 - 2010 based on Figure 4 for male population in which LM outperformed others, while for female population, HU6 is the most performing. 


\section{CONCLUSION}

This paper has compared and examined six methods based on principal component method for forecasting Malaysia ASDR. It is important to highlight that the period of study represents the only period for which data are available. It can be concluded that all methods predicted declining future deaths in almost all age groups for both genders when applied on Malaysia ASDR. It was found that the ability of the methods to produce good forecasts depends upon the number of historical data and number of steps in a step-ahead forecast. The performance of methods is also different for male and female populations. The LM method consistently outperform other methods for Malaysia male ASDR, especially for longer fitting period. All the methods did not consistently perform for Malaysia female ASDR. However, it seems that the LM and HU methods (HU1 and HU 6) are the best good of fit for Malaysia female ASDR. Since the data for this study is considerably short, it is important for further studies to consider the length of the period under study. However, it depends on the availability of Malaysia mortality data. In addition, an equivalent study should be conducted by using time series cross validation method in order to evaluate and validate the performance of the methods. This is due to the problem of the availability of short series for Malaysia mortality data.

\section{ACKNOWLEDGEMENTS}

The authors would like to thank Universiti Teknologi MARA (UiTM) for the facilities provided to conduct this study and the DOSM for providing the data on the number of deaths and population.

\section{REFERENCES}

[1] M. H. Omar, et al., "Healthy life expectancy vs health expenditure by sullivan method in Malaysia," Indonesian Journal of Electrical Engineering and Computer Science (IJEECS), vol 14, no. 1, pp. 402-406, 2019.

[2] R. D. Lee and L. R. Carter, "Modeling and Forecasting U.S. Mortality," Journal of the American Statistical Association, vol. 87, no. 419, pp. 659-671, 1992.

[3] N. M. N. Mathivanan et al., "A comparative study on dimensionality reduction between principal component analysis and k-means clustering," Indonesian Journal of Electrical Engineering and Computer Science (IJEECS), vol 16, no. 2, pp. 752-758, 2019.

[4] H. L. Booth, et al., "Evaluation of the variants of the Lee-Carter method of forecasting mortality: A multi-country comparison," New Zealand Population Review, vol. 31, no. 1, pp. 13-34, 2005.

[5] H. Booth, et al., "Lee-Carter mortality forecasting: A multi-country comparison of variants and extensions," Demographic Research, vol. 15, pp. 289-310, 2006.

[6] A. J. G. Cairns, et al., "Modelling and management of mortality risk: A review," Scandinavian Actuarial Journal, no. 2-3, pp. 79-113, 2008.

[7] R. D. Lee and T. Miller, "Evaluating the performance of the Lee-Carter method for forecasting mortality," Demography, vol. 38, no. 4, pp. 537-549, 2001.

[8] S. Tuljapurkar, N. Li, and C. Boe, "A universal pattern of mortality decline in the G7 countries," Nature, vol. 405, pp. 789-792, 2000.

[9] H. Booth, J. Maindonald and L. Smith, "Applying Lee-Carter under conditions of variable mortality decline," Population Studies, vol. 56, no. 3, pp. 325-336, 2002.

[10] R. J. Hyndman and M. Shahid Ullah, "Robust forecasting of mortality and fertility rates: A functional data approach," Computational Statistics \& Data Analysis, vol. 51, no. 10, pp. 4942-4956, 2007.

[11] H. L. Shang, H. Booth, and R. Hyndman, "Point and interval forecasts of mortality rates and life expectancy: A comparison of ten principal component methods," Demographic Research, vol. 25, pp. 173-214, 2011.

[12] K. Dowd et al., "Evaluating the goodness of fit of stochastic mortality models", Insurance: Mathematics and Economics, vol 47, no. 3, pp. 255-265, 2010.

[13] V. D'Amato, et al., "The mortality of the Italian population: Smoothing techniques on the Lee - Carter model," The Annals of Applied Statistics, vol. 5, no. 2A, pp. 705-724, 2011.

[14] C. Wang and Y. Liu, "Comparison of mortality modelling and forecasting - empirical evidence from Taiwan," International Research Journal of Finance and Economics, vol. 37, pp 46-55, 2010.

[15] N. Yasungnoen and P. Sattayatham, "Forecasting Thai mortality by using the Lee-Carter model," Asia-Pacific Journal of Risk and Insurance, vol. 10, no. 1, pp. 91-105, 2016.

[16] A. J. G. Cairns, et al., "Mortality density forecasts: An analysis of six stochastic mortality models," Insurance: Mathematics and Economics, vol. 48, no. 1, pp. 355-367, 2011.

[17] N. Ngataman, et al., "Forecasting the mortality rates of Malaysian population using Lee-Carter method," in Advances in Industrial and Applied Mathematics: Proceedings of 23rd Malaysian National Symposium of Mathematical Sciences (SKSM23), 2016.

[18] W. Z. W. Husin, et al., " Lee-Carter state space modeling: Application to the Malaysia mortality data" AIP Conference Proceedings, vol. 1602, no. 1, pp. 1002-1008, 2014.

[19] H. S. Kamaruddin and N. Ismail, "Forecasting selected specific age mortality rate of Malaysia by using Lee-Carter model," Journal of Physics: Conference Series, vol. 974, 2018. 
[20] W. Z. W. Husin, et al., "Evaluating the performance of the Lee-Carter method and its variants in modelling and forecasting Malaysia mortality," AIP Conference Proceedings, vol. 1635, no. 1, pp. 762-769, 2014.

[21] W. Z. W. Husin, et al., "Stochastic models in forecasting Malaysia mortality: The Lee-Carter model and its extension," Advanced Science Letters, vol. 21, no. 6, pp. 1850-1853, 2015.

[22] H. S. Kamaruddin, "Forecasting mortality rate of Malaysia using Lee-Carter Extension of Hynman-Ullah and Booth-Maindonald-Smith and its statistical comparison analysis," The 4th Abu Dhabi University Annual International Conference: Mathematical Science \& Its Applications, 2015.

[23] S. N. Shair, et al., "A Functional data approach to the estimation of mortality and life expectancy at birth in developing countries," Pertanika Journal of Science \& Technology, vol. 27, no. 2, pp. 797-814, 2019.

[24] H. S. Kamaruddin and N. Ismail, "Statistical comparison of projection Malaysia mortality rate by using Lee-Carter model and Lee-Carter extension of Hyndman-Ullah," AIP Conference Proceedings, vol. 2111, no. 1, 2019.

[25] N. S. M. Ibrahim et al., "Mortality rates and life expectancy improvements among Malaysian elderlies," Indonesian Journal of Electrical Engineering and Computer Science (IJEECS), vol 19, no. 1, pp. $134-139,2020$

[26] E. M. Crimmins et al., "Differences between men and women in mortality and the health dimensions of the morbidity process," Clinical chemistry, vol. 65, no. 1, pp. 135-145, 2019.

[27] R. J. Hyndman and H. Booth, "Stochastic population forecasts using functional data models for mortality, fertility and migration," International Journal of Forecasting, vol. 24, no. 3, pp. 323-342, 2008.

\section{BIOGRAPHIES OF AUTHORS}

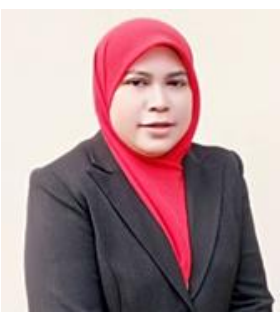

Dr. Wan Zakiyatussariroh Wan Husin holds a $\mathrm{PhD}$ in Statistics from Universiti Teknologi MARA, MSc in Applied Statistics from Universiti Putra Malaysia and a BSc Hons in Statistics also from Universiti Teknologi MARA. She is currently working in Universiti Teknologi MARA as a Senior Lecturer and has several years of working experience in the areas of teaching, research and administration. Dr. Wan Zakiyatussariroh has several research publications in wellknown international journals and conferences. She received the Best Potential Research for Commercialization Award in 2010, which was awarded by Universiti Teknologi MARA. She also won the Best Paper Award in CSSR2019. She has also been engaged to create linkages between industry and academia. She is a member of the Malaysian Institute of Statistics, the ASEAN Population Association (APA), the International Association of Engineers (IAENG) as well as the Management Science and Operations Research Society of Malaysia. Her research interests include Statistical Demography, Time Series and Forecasting Modelling and Optimization Modelling. She is currently conducting a research on Dynamic State Space SpatioTemporal Forecasting Model for Under-5 Mortality.

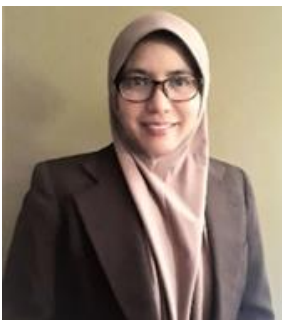

Nazatul Shahreen Zainal Abidin holds an MA (TESL) from Universiti Kebangsaan Malaysia and a BA in Economics from Acadia University, Canada. She is currently working in Universiti Teknologi MARA Kelantan branch as a Senior Lecturer and has 18 years of experience in the areas of language teaching and research. She has several research publications in international journals and conferences. Her research interests include second language acquisition, specifically task-based language learning and teaching and the contribution of individual differences in learning through oral interaction (e.g. working memory, language aptitude, motivation, learning preferences). 